\title{
Eficácia da clorexidina-cetrimida na desinfecção ambiental contra Aspergillus spp.
}

\author{
[Chlorexidine-cetrimide efficacy in ambient disinfection against Aspergillus spp.]
}

\author{
M.O. Xavier $^{1}$, A.R.M. Meinerz ${ }^{2}$, M.B. Cleff $^{2}$, L.G. Osório ${ }^{1}$, L.F.D. Schuch ${ }^{2}$, M.O. Nobre ${ }^{1}$, \\ R.P. Silva Filho ${ }^{3}$, M.C.A. Meireles ${ }^{1}$ \\ ${ }^{1}$ Faculdade de Veterinária - UFPel - Pelotas, RS \\ ${ }^{2}$ Faculdade de Veterinária - UFRGS - Porto Alegre, RS \\ ${ }^{3}$ Centro de Recuperação de Animais Marinhos - FURG - Rio Grande, RS
}

\begin{abstract}
RESUMO
Avaliou-se a ação in loco da clorexidina-cetrimida no controle de Aspergillus spp., considerando-se a influência de fatores climáticos e populacionais, em um centro de recuperação de animais marinhos. Durante dois anos, realizaram-se colheitas de amostras de ar por meio da técnica de sedimentação, com implantação no segundo ano de um programa de desinfecção com clorexidina-cetrimida. Os resultados do isolamento fúngico nos dois anos foram comparados estatisticamente pelo programa Epinfo 8.0. Demonstrou-se que o isolamento de Aspergillus spp. não apresentou relação significativa com fatores climáticos e populacionais e que a diminuição da concentração de conídios de Aspergillus spp. do ambiente ocorreu devido ao programa eficaz de desinfecção com a clorexidina-cetrimida.
\end{abstract}

Palavras-chave: Aspergillus spp., clorexidina-cetrimida, desinfetante

\begin{abstract}
The study evaluated the in loco activity of chlorexidine-cetrimide for environmental control of Aspergillus spp. considering the climatic influence and population variations, in a recuperation center of marine animals. Samples of air were collected throughout two years by the sediment method, and the disinfection program was established in the second year. Data collected during the two years were statistically compared by Epinfo 8.0. program. It was demonstrated that both population and climate had no influence on the fungal isolation, and the disinfection program with chlorexidine-cetrimide was considered effective in the reduction of conidia Aspergillus concentration indoor.
\end{abstract}

Keywords: Aspergillus spp., chlorexidine, disinfectant

\section{INTRODUÇÃO}

Os programas de higiene e desinfecção são essenciais no controle ambiental de microrganismos potencialmente patogênicos aos animais. Dentre esses patógenos, em relação a aves em cativeiro, destacam-se os fungos anemófilos e ubíquos do gênero Aspergillus, que causam uma micose respiratória geralmente fatal conhecida como aspergilose (Redig et al., 1993; Garcia e Blanco, 2000; Lagneau e Houtain, 2001).

Recebido em 14 de setembro de 2007

Aceito em 18 de julho de 2008

Endereço para correspondência (corresponding address)

Rua Paul Harris, 537 - 96077-200 - Pelotas, RS

E-mail: melissaxavier@bol.com.br
Os fungos do gênero Aspergillus reproduzem-se por meio de fialoconídios pequenos e leves, facilmente veiculados pelo ar, que se depositam em qualquer objeto ou superfície e contaminam todo o ambiente (Latgé, 1999; Warris e Verweij, 2005). Assim, para o controle da aspergilose, é preconizada a manutenção correta da ventilação, da temperatura e da umidade do local, bem como a higiene e a desinfecção ambiental rotineira (Kearns e Loudis, 2003; Warris e Verweij, 2005). 
A desinfecção ambiental deve ser realizada com rodízio periódico de agentes químicos capazes de inibir o crescimento fúngico ou eliminar esses microrganismos, visando à redução na concentração de conídios inalados pelas aves (Kearns e Loudis, 2003). Dentre os agentes desinfetantes, as biguanidas, como a clorexidina, se destacam principalmente pela baixa toxicidade, amplo espectro e suas propriedades inodoras, incolores e não corrosivas (McDonnell e Russell, 1999).

Tendo em vista que a aspergilose é uma das principais causas de mortalidade de pingüins em cativeiro (Ainsworth e Rewell, 1949; Flach et al., 1990; Xavier et al., 2006) e considerando a presença dessas aves marinhas no local da desinfecção, assim como a importância na prevenção da micose em centros de reabilitação, este trabalho teve por objetivo testar a ação da Clorexidina-Cetrimida ${ }^{\circledR}{ }^{1}$, no controle de Aspergillus spp. em instalações do Centro de Recuperação de Animais Marinhos (CRAM), avaliando a influência dos fatores climáticos e populacionais no isolamento fúngico.

\section{MATERIAL E MÉTODOS}

Durante dois anos, foi avaliada a qualidade do ar, quanto à presença de Aspergillus spp., e das instalações internas utilizadas para reabilitação de pingüins no CRAM, localizado no município de Rio Grande, RS. Essas instalações eram caracterizadas por dois ambientes de $12 \mathrm{~m}$ de comprimento, $4 \mathrm{~m}$ de largura e $4 \mathrm{~m}$ de altura, com paredes azulejadas e portões grandes $(2 \times 2 \mathrm{~m})$ nas duas extremidades, os quais permaneciam abertos durante os turnos de trabalho, permitindo o fluxo de ar, para manutenção da ventilação do local.

As amostras do ar foram colhidas pela técnica de sedimentação, realizada a partir da exposição por 15 minutos, de placas de Petri contendo ágar Sabouraud dextrose acrescido de cloranfenicol. A colheita foi realizada em intervalos de 10 dias no primeiro ano e semanalmente no segundo ano do experimento, coletando-se uma amostra referente ao horário de abertura do CRAM (8h) e uma segunda amostra no final do expediente matinal (11h30min) após higienização e ventilação natural do local.

${ }^{1}$ Chemitec Agro-Veterinária, São Paulo, Brasil.
As placas eram imediatamente incubadas em estufa a $25^{\circ} \mathrm{C}$ durante sete dias e observadas diariamente. As colônias fúngicas filamentosas foram avaliadas macro e micromorfologicamente para identificação taxonômica, sendo realizada subcultura em ágar Czapek para obtenção da cultura pura dos isolados de Aspergillus spp.

No segundo ano do experimento, foi realizado um programa de desinfecção das instalações a partir do emprego da Clorexidina-Cetrimida ${ }^{\circledR}$ diariamente, nos locais de contaminação por matéria orgânica, e, semanalmente, em todos os objetos e superfícies, incluindo paredes e teto. A desinfecção era realizada ao encerramento das atividades diárias, após limpeza prévia e retirada da matéria orgânica, por meio da utilização, por fricção mecânica do produto comercial, em diluição indicada pelo fabricante $(100 \mathrm{ml} / 1 \mathrm{de}$ água), e posterior enxágüe com água corrente. Logo em seguida, o ambiente era mantido fechado por um período de aproximadamente 14 horas, até a manhã do dia seguinte.

A densidade populacional do local no momento das colheitas das amostras foi obtida junto ao CRAM, e os dados meteorológicos da cidade de Rio Grande, RS, como temperatura média mensal, umidade relativa do ar média mensal e precipitação total mensal, foram gentilmente cedidos pelo $8^{\circ}$ Distrito de Meteorologia.

Os resultados foram analisados estatisticamente pelo teste de qui-quadrado por meio do programa Epinfo 8.0.

\section{RESULTADOS E DISCUSSÃO}

Ao final do experimento, o número total de amostras colhidas foi $160 ; 64$ relativas ao primeiro ano e 96 ao segundo ano do estudo, com resultado positivo para Aspergillus spp. em 22,5\% (36) das amostras; dessas, 17 eram do primeiro ano e 19 do segundo ano (Tab. 1).

O pequeno número de isolados de Aspergillus spp. observado no experimento pode ser atribuído ao método utilizado para colheita das amostras, cujo princípio se baseia na ação da gravidade do ar, sofrendo influência da velocidade e turbulência do vento que podem carrear os propágulos fúngicos, dificultando que se depositem na placa (Morris et al., 2000). 
Eficácia da clorexidina-cetrimida...

Tabela 1. Isolamento de fungos do gênero Aspergillus de amostras de ar colhidas durante dois anos nas instalações utilizadas para reabilitação de pingüins

\begin{tabular}{|c|c|c|c|c|}
\hline Amostras & $\begin{array}{c}\text { Positivas } \\
8 \mathrm{~h}\end{array}$ & $\begin{array}{l}\text { Positivas } \\
11 \mathrm{~h} 30 \mathrm{~min}\end{array}$ & Negativas & Total de amostras \\
\hline $1^{\circ}$ ano & $14(21,9 \%)$ & $3(4,7 \%)$ & $47(73,4 \%)$ & 64 \\
\hline $2^{\circ}$ ano & $10(10,4 \%)$ & $9(9,4 \%)$ & $77(80,2 \%)$ & 96 \\
\hline Total & $24(15,0 \%)$ & $12(7,5 \%)$ & $124(77,5 \%)$ & 160 \\
\hline
\end{tabular}

Durante os dois anos do estudo, a temperatura média mensal variou entre $13^{\circ} \mathrm{C}$ e $24,2^{\circ} \mathrm{C}$, a umidade relativa do ar média entre 68 e $91 \%$ e a precipitação média mensal entre 17,2 e $355,1 \mathrm{~mm}$ (Fig. 1, 2). Apesar da grande variação dos dados, os três fatores climáticos não apresentaram relação significativa com o isolamento de Aspergillus spp. das instalações. Obtiveram-se valores estatísticos de $\mathrm{P}=0,996$ para temperatura, $\mathrm{P}=0,794$ para umidade $\mathrm{e}$ $\mathrm{P}=0,224$ para precipitação.

A relação não significativa entre temperatura e isolamento fúngico já foi descrita previamente por diversos autores (Panagopoulou et al., 2002; Wu et al., 2005; Lee et al., 2006), assim como a não significância da precipitação em amostras positivas (Lee et al., 2006). A influência da umidade no isolamento fúngico tem resultados contraditórios entre os estudos. Alguns autores acreditam que exista uma relação (Lee et al., 2006), enquanto outros demonstraram que esse fator não é determinante para o isolamento fúngico (Ren et al., 2001; Panagopoulou et al., 2002; Wu et al., 2005).
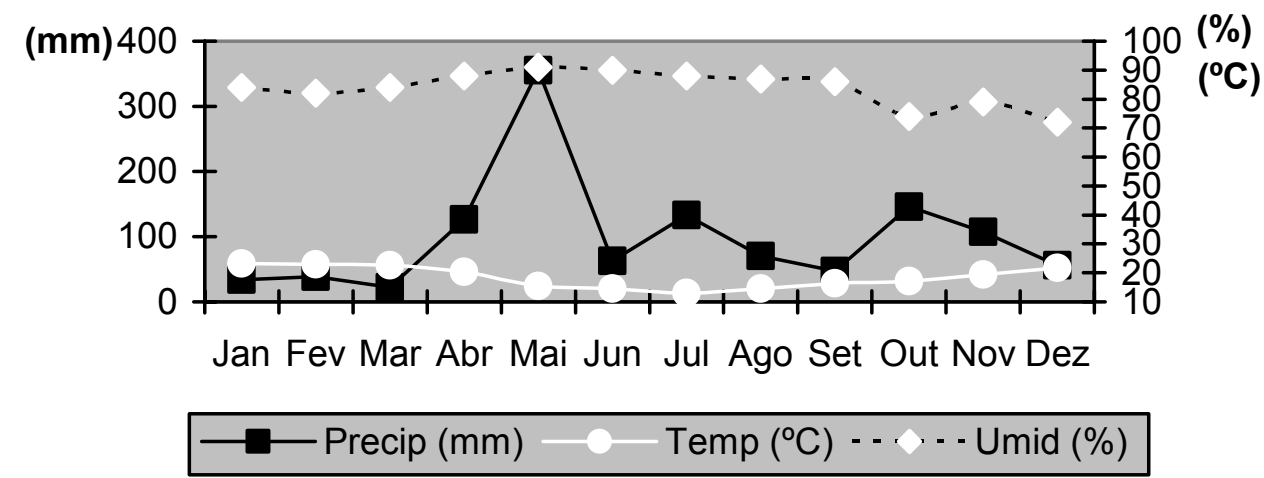

Figura1. Variação climática de temperatura $\left({ }^{\circ} \mathrm{C}\right)$, umidade $(\%)$ e precipitação $(\mathrm{mm})$ da cidade de Rio Grande no primeiro ano do experimento.

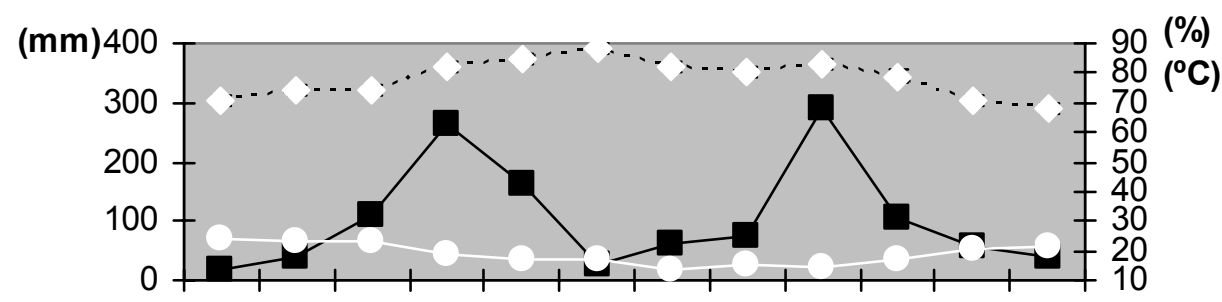

Jan Fev Mar Abr Mai Jun Jul Ago Set Out Nov Dez

$\longrightarrow$ Precip $(\mathrm{mm}) \longrightarrow \operatorname{Temp}\left({ }^{\circ} \mathrm{C}\right) \cdots \cdots$ Umid $(\%)$

Figua 2. Variação climática, de temperatura $\left({ }^{\circ} \mathrm{C}\right)$, umidade $(\%)$ e precipitação $(\mathrm{mm})$ da cidade de Rio Grande no segundo ano do experimento. 
O isolamento de Aspergillus spp. no segundo ano foi significativamente menor $(\mathrm{P}=0,025$; considerando $\mathrm{P}<0,05)$ nas colheitas das 8 horas em relação ao ano inicial, enquanto nas amostras das 11h30min não houve diferença significativa $(\mathrm{P}=0,168)$ entre os dois anos.

Tendo em vista que fatores climáticos e densidade populacional não interferiram no isolamento de Aspergillus spp. do ambiente, a diminuição significativa dos isolados na primeira colheita da manhã foi atribuída ao programa de desinfecção empregado, que conseguiu minimizar a proliferação fúngica durante $o$ período crítico, em que o CRAM permanecia fechado, após expediente de trabalho interno diário. Outros estudos da ação in loco desse agente químico frente a fungos anemófilos, como o Aspergillus spp., não foram encontrados na literatura, no entanto testes in vitro da ação antifúngica do digluconato de clorexidina demonstraram resultados positivos contra fungos do gênero Aspergillus (Xavier et al., 2007).

Agentes químicos à base de digluconato de clorexidina são amplamente utilizados na prática odontológica e na antissepsia, porém sua aplicação como desinfetante ambiental é limitada. Neste experimento, esse agente químico foi escolhido principalmente devido à necessidade de aplicação em instalações com a presença de animais, tendo em vista que os produtos mais freqüentemente utilizados para desinfecção de ambientes internos, como hipoclorito de sódio e amônia quaternária, são irritantes para mucosas e pele, e a amônia quaternária ainda é passível de contaminação, podendo carrear e disseminar microrganismos para o ambiente (McDonnell e Russell, 1999).

Nas colheitas das 11h30min não ocorreu redução do isolamento fúngico entre os dois anos estudados, provavelmente devido à ventilação natural utilizada no CRAM, que permite a veiculação de propágulos fúngicos do ar externo para o interior das instalações, recontaminando o ambiente. Esse fato também foi observado em estudos envolvendo ambientes hospitalares, residências e prédios comerciais, onde vários autores comprovaram que o ar externo é a maior fonte de contaminação fúngica para as instalações internas (Leenders et al., 1999; Shelton et al., 2002; Lee et al., 2006).

\section{CONCLUSÃO}

O uso da Clorexidina-Cetrimida ${ }^{\circledR}$ em programas de desinfecção ambiental demonstrou eficácia no controle de fungos do gênero Aspergillus, podendo ser utilizada in loco para desinfecção de instalações de cativeiro de pingüins, auxiliando na prevenção da aspergilose.

\section{REFERÊNCIAS BIBLIOGRÁFICAS}

AINSWORTH, G.C.; REWELL, R.E. The incidence of aspergillosis in captive wild birds. $J$. Comp. Path. Therap., v.59, p.213-224, 1949.

FLACH, E.J.; STEVENSON, M.F.; HENDERSON, G.M. Aspergillosis in gentoo penguins (Pygoscelis papua) at Edinburgh Zoo, 1964-1988. Vet. Rec., v.126, p.81-85, 1990.

GARCÍA, M.E.; BLANCO, J.L. Principales enfermedades fúngicas que afectan a los animales domésticos. Rev. Iberoam. Micol., v.17, p.2-7, 2000.

KEARNS, K. S.; LOUDIS B. Aspergillosis aviar. In: Recent Advances in Avian Infectious Diseases. Ithaca, NY: International Veterinary Information Service, 2003.

LAGNEAU, P.E.; HOUTAIN, J.Y. Aspergillose invasive chez des psittacidés. Ann. Med. Vet., v.145, p.307-310, 2001.

LATGÉ, J.P. Aspergillus fumigatus and Aspergillosis. Clin. Microbiol. Rev., v.12, p.310350, 1999.

LEE, T.; GRINSHPUN, S.A.; MARTUZEVICIUS, D. et al. Relationship between indoor and outdoor bioaerosols collected with a button inhalable aerosol sampler in urban homes. Indoor Air, v.16, p.37-47, 2006.

LEENDERS, A.C.A.P.; BELKUM, A.V.; BEHRENDT, M. et al. Density and molecular epidemiology of Aspergillus in air and relationship to outbreaks of Aspergillus infection. J. Clin. Microbiol., v.37, p.1752-1757, 1999.

MCDONNELL, G.; RUSSEL, A.D. Antiseptics and disinfectants: activity, action, and resistance. Clin. Microbiol. Rev., v.12, p.147-179, 1999.

MORRIS, G.; KOKKI, M.H.; ANDERSON, K. et al. Sampling of Aspergillus spores in air. $J$. Hosp. Inf., v.44, p.81-92, 2000. 
PANAGOPOULOU, P.; FILIOTI, J.; PETRIKKOS, G. et al. Environmental surveillance of filamentous fungi in three tertiary care hospitals in Greece. J. Hosp. Inf., v.52, p.185-191, 2002.

REDIG, P.T. General infectious diseases: avian aspergillosis. In: FOWLER, M.E.; MILLER, R.E. (EdS). Zoo \& Wild Animal Medicine: current therapy 3, Denver: Elsevier, 1993. p.178181.

REN, P.; JANKUN, T.M.; BELANGER, K. et al. The relation between fungal propagules in indoor air and home characteristics. Allergy, v.56, p.419-424, 2001.

SHELTON, B.G.; KIRKLAND, K.H.; FLANDERS, W.D. et al. Profiles of Airborne Fungi in Buildings and Outdoor Environments in the United States. Appl. Envir. Microbiol., v.68, p.1743-1753, 2002.
XAVIER, M.O.; LEITE, A.T.M.; SOARES, M.P. et al. Aspergilose em pingüim-demagalhães (Spheniscus magellanicus) - Relato de caso. Vet. Zootec., v.13, p.28-32, 2006.

XAVIER, M.O; MADRID, I.M.; MEINERZ, A.R.M. et al. Atividade in vitro de três agentes químicos frente a diferentes espécies de Aspergillus. Arq. Inst. Biol., v.74, p.49-53, 2007.

WARRIS, A.; VERWEIJ, P.E. Clinical implications of environmental sources for Aspergillus. Med. Mycol., v.43, p.59-65, 2005.

WU, P.C.; LI, Y.Y.; CHIANG, C.M. et al. Changing microbial concentrations are associated with ventilation performance in Taiwan's air-conditioned office buildings. Indoor Air, v.15, p.19-26, 2005. 